\title{
Can Laplacian Eigenmaps Be Used for Differentiation Between Healthy Subjects and Patients With Corrected Tetralogy of Fallot?
}

\author{
Ben Jacobs $^{1}$, Amalia Villa ${ }^{1}$, Jonathan Moeyersons ${ }^{1}$, \\ Sabine Van Huffel ${ }^{1}$, Rik Willems ${ }^{2}$ and Carolina Varon ${ }^{3}$ \\ ${ }^{1}$ KU Leuven, Department of Electrical Engineering (ESAT), STADIUS, Leuven, Belgium \\ ${ }^{2}$ Division of Experimental Cardiology, Department of Cardiovascular Diseases, KU Leuven, Belgium \\ ${ }^{3}$ Circuits and Systems (CAS) group, Delft University of Technology, The Netherlands
}

\begin{abstract}
Tetralogy of Fallot (ToF) is a congenital structural heart disease. While early diagnosis and corrective surgery allow most patients to live normal lives, some patients slowly deteriorate. The current inability to quantify the deterioration and predict these events prompts a data driven approach. Laplacian Eigenmaps (LEs) are a dimensionality reduction technique that can be used to project multi-lead ECGs onto a lower dimensional space. This pilot study aims to evaluate the ability of LEs to characterize deterioration of ToF patients.

A general LE model is constructed, based on the 12-lead ECG recordings of 20 healthy controls. A set of distance metrics are developed to quantify the overall changes between different ECG recordings within this LE model.

Statistically significant differences between control and ToF subjects were observed for most of the distance metrics. The analysis of changes over time in ToF patients indicates a general trend of increased distance over time in all the metrics, which can be related to a worsening condition. This indicates the relevance of LEs in multi-lead ECG processing, particularly for deterioration analysis.
\end{abstract}

\section{Introduction}

Tetralogy of Fallot (ToF) is the most prevalent cyanotic congenital heart defect. The survival rate of ToF patients has improved drastically since surgical correction shortly after birth became the preferred form of treatment. However, an increasing number of adults with corrected ToF present late complications such as arrhythmias, heart failure and sudden cardiac death. These complications are the effects of long-term pulmonary regurgitation and consequently increased fibrosis in the right ventricle. Initially the heart is able to compensate for this, but after years of overload and deterioration, it can fail [1].

Longitudinal analysis of ECG recordings of corrected ToF patients can be used to characterize the slow dete- rioration of their heart. Laplacian Eigenmaps (LE) is a machine learning technique that can assist this analysis by representing 12-lead ECG data into a lower dimensional space [2]. This framework captures and emphasises local differences in the data. Good et al. [3] obtained promising results when using this technique to quantify induced ischemia in animal subjects.

This work serves as a pilot study with the overall aim to investigate the possibilities of LEs for longitudinal ECG analysis of corrected ToF patients. The hypothesis presented is that the ability of the LEs to highlight local differences makes it suitable to track small general changes over time in multi-lead ECG data.

This overall aim is addressed with two concrete objectives. The first objective is to create a general LE model to which multiple ECGs can be mapped and compared. The second is to quantify the overall changes between different ECG recordings within this model.

\section{Datasets}

The dataset of corrected ToF patients consists of longitudinal 12-lead ECG measurements of 100 patients recorded in UZ Leuven. Most of these patients visit the hospital for a yearly check-up. Out of these 100 patients, 6 died of cardiac reasons. These are the patients considered in the presented study, since this group of patients can be assumed to have a comparable level of fibrosis in their last visit.

The WFDB dataset from the PhysioNet/Computing in Cardiology Challenge 2020 [4] is used in these experiments as a source of control patients. It consists of 12lead ECG measurements of 6877 subjects with healthy and deviating heart rhythms, obtained from multiple hospitals. Only the 918 healthy recordings are further utilised.

\section{Constructing the General LE model}

The general LE model can be interpreted as a reference space, further called the normal space, to which 12-lead 


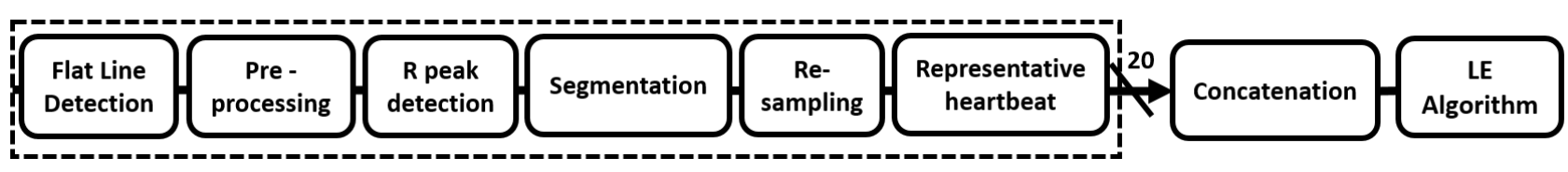

Figure 1. Schematic for constructing the normal space

ECG recordings can be mapped. It is constructed based on 20 random 12-lead ECGs of healthy control subjects from the WFDB dataset. The different steps needed for the implementation of the normal space are depicted in Figure 1.

Flat line detection is necessary to ensure that no erroneous leads were included in the analysis. If one or more of the 12 leads are missing, the complete recording is discarded. The pre-processing step filters the raw signal with a fourth order Butterworth bandpass filter with lower and upper cut-off frequencies of 0.67 and $30 \mathrm{~Hz}$ respectively [5].

After filtering, an R peak detection algorithm is applied to lead I [6]. Only normal heartbeats are withheld for analysis, hence an ectopic beat removal step based on RR intervals is applied to the detected $\mathrm{R}$ peaks.

Based on the locations of the R peaks, the 12-lead ECG recordings are segmented in separate heartbeats. In order to do so, the interval between two consecutive $\mathrm{R}$ peaks is split into two equal pieces assigned to the adjacent heartbeats. This segmentation technique allows to incorporate the maximum amount of information in the ECG. Each segmented heartbeat is then resampled to $200 \mathrm{~Hz}$.

In order to synthesize the information from each patient and make the model more robust, only the most representative heartbeat of the complete recording is selected. This selection happens fully automatic based on the crosscorrelation between heartbeats.

All the different steps mentioned before are repeated for the 20 healthy control subjects who will conform the normal space. The 12-lead ECGs of each of the 20 representative heartbeats are concatenated into one signal. This number of heartbeats allows the model to be based on a heterogeneous set of heartbeats while keeping a low computation time.

The selected heartbeats are fed to the LE algorithm, which is a nonlinear dimensionality reduction technique based on graph theory. It allows to capture the underlying structures in high-dimensional data, into a lower dimensional space. It consists of applying a radial basis function (RBF) to all the points of the concatenated signal. As a result, a similarity matrix $\mathbf{W}$ is computed, which emphasizes local differences in the signal. The underlying structures in the data are then derived using singular value decomposition (SVD):

$$
\mathbf{D}^{-1} \mathbf{W}=\mathbf{U S V}^{-1}
$$

(A) Controls

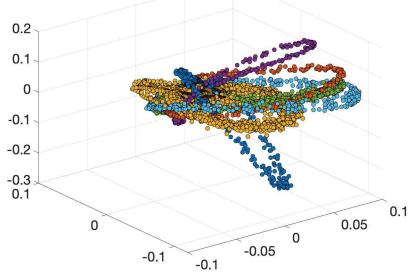

(B) ToF patients

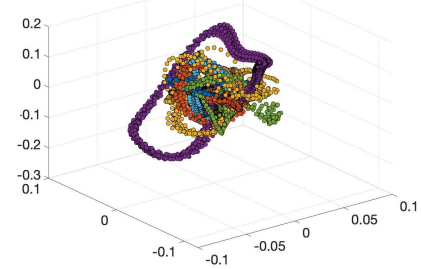

Figure 2. Normal space: (A) The LEs of six control subjects mapped onto the normal space. (B) The last visit of six ToF patients who died of cardiac events, mapped onto the normal space.

where $\mathbf{D}$ is the degree matrix of $\mathbf{W}$. SVD derives a transformation that maps the points of the signal onto a new space, where the amount of information is ranked along the coordinates.

It is assumed that most of the information is contained in the singular vectors corresponding to the highest singular values, after excluding the largest one [2]. Therefore, the "normal space" is defined as:

$$
L E s=\left[\mathbf{V}_{\mathbf{2}}, \mathbf{V}_{\mathbf{3}}, \mathbf{V}_{\mathbf{4}}\right]
$$

In machine learning terms, the construction of the normal space can be understood as the training phase. In the mapping phase, the transformation obtained from the SVD can be used to map any 12-lead ECG signal onto the normal space with a process called explicit mapping [2]. The same steps as in Figure 1 need to be applied to the ECG of a subject, except for the representative heartbeat selection. In this case, the concatenated signal consists of multiple heartbeats of the same recording. Each heartbeat is represented by a separate LE loop in the normal space, as shown in Figure 2.

\section{Distance Measures}

Since an ECG recording of a patient, further referred to as a visit, mapped onto the normal space consists of multiple loops, the average of those loops is taken to represent the visit. A set of distance measures is proposed to analyze the global differences between two average LE loops.

- Point-to-point Distance: It calculates the euclidean distance between the corresponding points of two average LE loops, given that they are adjusted to the same length. A 
single value is obtained by summing over all the points of the loop.

- Hausdorff Distance: This is a frequently used metric that expresses how similar two sets of points are [7]. It achieves this by searching for one point in both sets (i.e. loop) with the furthest euclidean distance to its nearest neighbor. This is the Hausdorff distance.

- Dynamic Time Warping Distance (DTW): DTW is an algorithm used to align two time series. It looks for an optimal alignment between the signals, by stretching them in order to minimise the total euclidean distance between them [8].

- Eigenshape distance: The eigenshape distance metric is a method based on a technique frequently used in medical imaging [9]. This algorithm uses principal component analysis (PCA) to construct an eigenshape model of a general LE loop. This model consists of an average LE loop and six modes of variation, called eigenshapes. Other LE loops can then be expressed as instances of this model by a linear combination of the eigenshapes. The associated coefficients are translated into a distance value that correlates with how much the loop deviates from the model.

\section{Experiments}

\subsection{Compare ToF Patients and Controls}

This experiment compares the last visit of the six ToF patients who died of cardiac events to six recordings from control patients. The visits of both groups were mapped onto the normal space (Figure 2). The distance measures were used to calculate the difference between the average loop of a subject and a reference loop, called the standard loop. The latter was constructed as the average of the loops of the 20 controls that were initially used to build the normal space. The eigenshape distance does not use the standard loop, but it alternatively creates an eigenshape model based on the the loops of the 20 controls. The average loop of the analyzed subject is then expressed as an instance of this model.

The complete experiment was repeated 10 times. In each of the 10 folds, 20 controls were randomly selected to construct the normal space and the standard loop. Six other controls were randomly selected to be compared against the ToF patients. A Kolmogorov-Smirnov test was used to compare the patients and control group, and $p<0.05$ was considered significant. The hypothesis was that the average distances of the control subjects are closer to the standard loop than those of the patients.

\subsection{Evolution over time}

The second experiment investigated the changes in the loops of the patients over time. Five of the six patients

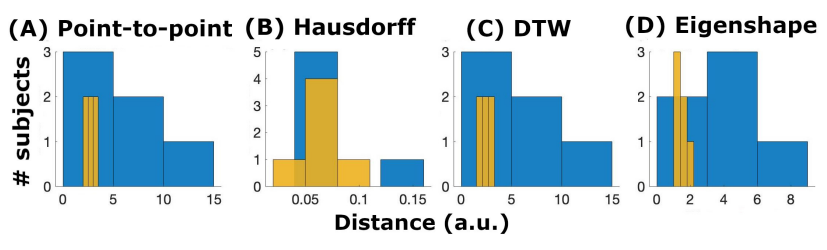

Figure 3. Distance measures for patients (blue) and controls (yellow), for 1 fold: The point-to-point distance (A), DTW distance $(C)$ and eigenshape distance (D) are in general larger and depict a wider spread in the patient group.

were included, since one of them had only two ECG recordings available. Additionally, to compensate for different deterioration rates of the patients, the amount of visits included in the analysis were limited to five: the last visit before death and the last visit of each year for the previous four years. All the distance measures were calculated relative to the average loop of the first included visit of the patient. For the eigenshape distance, the model was constructed on that same visit and the average loops of later visits were expressed as instances of this model. Since this model is unique to every patient, so are the corresponding distance values. Therefore, they were normalized before comparing the trends between different patients. The distance measures were expected to depict an upwards trend over time.

\section{Results and Discussion}

\subsection{Compare ToF Patients and Controls}

The results of the individual folds were very similar. One fold is given as an example in Figure 3. Statistically significant differences were observed in 10/10, 2/10, 10/10 and $7 / 10$ folds for the point-to-point, Hausdorff, DTW and eigenshape distance, respectively.

For all folds, the mean of the distance measures in the control group was lower than in the ToF patients. This confirms the initial hypothesis. Additionally, the spread is much larger in the results of the patients, suggesting that they present different levels of fibrosis at their last visit.

Although the point-to-point and DTW distance were able to distinguish between both groups in all the folds, the DTW distance can generally be expected to deliver better results since it is more robust against the misalignment of the segments. However, the absolute values of the pointto-point and DTW distance are very similar to each other in this case. This indicates that not much alignment between the average loops and the standard loop is needed before an optimum is reached in the DTW algorithm.

The Hausdorff distance scored worse than the other distance metrics. This is due to its sensitivity to outliers.

The eigenshape distance was slightly outperformed by 
(A) Point-to-point

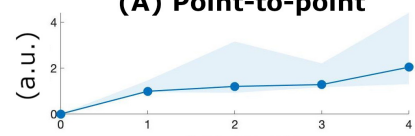

(C) DTW

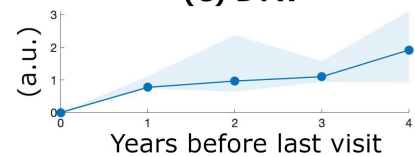

Yearrs before last visit

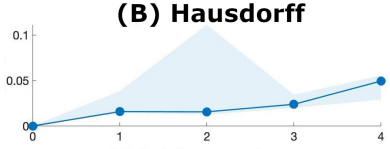

(D) Eigenshape

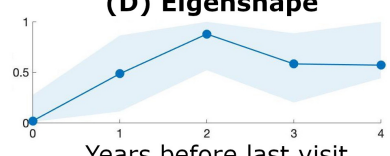

Years before last visit

Figure 4. Evolution over time of the distance metrics relative to the first visit (year zero). The blue line indicates the average. The upper and lower boundary represent the 25 th and 75 th percentile respectively.

the point-to-point and DTW distance. This can be explained by the amount of loops used for the construction of the eigenshape model. Including more loops incorporates more flexibility in the model to reproduce more unfamiliar shapes. However, this comes with a high computational cost related to the application of the kernel.

\subsection{Evolution over time}

The initial hypothesis expecting an upwards trend over time in the distance measures is confirmed by the results shown in Figure 4. These show that the normal space in combination with the distance measures are capable of capturing the deterioration in the patients, since the ToF ECG segments show increasing differences over time.

In the eigenshape distance, this trend was less pronounced, but the same limitations as in the previous experiment applied.

In contrast to the work of Good et al. [3], who researched the effect of increased ischemic stress with LEs by focusing on one particular point along the loops, the distance metrics in this work incorporate information from all the points of the loops to obtain a more general measure. This results in a loss of specificity, but offers a more general analysis of the deterioration of the patients.

This work opens the path to apply LE to longitudinal ECG data, allowing to find local changes between consecutive visits. Future research should explore the use of this approach for specific parts of the ECG, in order to retrieve more specific conclusions. Additionally, longitudinal analysis should also be performed on control data, which was unavailable in this study.

\section{Conclusion}

This work serves as a proof of concept for the application of LEs on 12-lead ECG data to track the general deterioration of corrected ToF patients. This was confirmed in two different ways. First by creating a normal space from control ECG data where healthy and ToF ECGs can

be compared using a set of 3D distance measures. Significant differences were found between both groups. Additionally, the longitudinal analysis of ToF ECGs in this normal spaced showed an increase in these distances that can be related to deterioration. The promising results of this pilot study suggest that the general LE model in combination with the distance measures can be applied in future research for other longitudinal applications.

\section{Acknowledgments}

Research funded by Agentschap Innoveren en Ondernemen (VLAIO) 150466: OSA+. KU Leuven Stadius acknowledges the financial support of imec. This research received funding from the Flemish Government (AI Research Program). SVH, AV and CV affiliated to Leuven.AI - KU Leuven institute for AI, B-3000, Leuven, Belgium. R. Willems is supported as postdoctoral clinical researcher by the Fund for Scientific Research Flanders.

\section{References}

[1] Dłużniewska N, et al. Long-term follow-up in adults after Tetralogy of Fallot repair. Cardiovascular Ultrasound 2018;

[2] Erem B, et al. Extensions to a manifold learning framework for time-series analysis on dynamic manifolds in bioelectric signals. Phys Rev E 2016;

[3] Good W, et al. Temporal performance of laplacian eigenmaps and 3D conduction velocity in detecting ischemic stress. Journal of Electrocardiology 2018;

[4] Classification of 12-lead ECGs: the physionet/computing in cardiology challenge 2020. https://physionetchallenges.github.io/2020/. Accessed: 2020-06-04.

[5] Kligfield P, et al. Recommendations for the standardization and interpretation of the electrocardiogram. Part I: the electrocardiogram and its technology, a scientific statement. Journal of the American College of Cardiology 2007; 49(10):1109-1127.

[6] Moeyersons J, et al. R-deco: An open-source matlab based graphical user interface for the detection and correction of R-peaks. PeerJ Computer Science 2019;

[7] Delfour M, et al. Shapes and geometries: metrics, analysis, differential calculus, and optimization. Society for Industrial and Applied Mathematics, 2011.

[8] Muller M, et al. Information Retrieval for Music and Motion. Springer, Berlin, Heidelberg, 2007;

[9] Suetens P. Fundamentals of Medical Imaging. Cambridge University Press, 2009; .

Address for correspondence:

Amalia Villa

Department of Electrical Engineering (ESAT),

STADIUS, KU Leuven, Belgium

amalia.villagomez@kuleuven.be 